\title{
Locally compact groups which are separably categorical structures
}

\author{
Aleksander Ivanov ${ }^{1,2}$
}

Received: 31 May 2015 / Accepted: 26 October 2016 / Published online: 3 November 2016

(C) The Author(s) 2016. This article is published with open access at Springerlink.com

\begin{abstract}
We describe locally compact groups which are separably categorical metric structures.
\end{abstract}

Keywords Separably categorical structures $\cdot$ Locally compact groups $\cdot$ Stability

Mathematics Subject Classification 03C60

\section{Introduction}

In this paper we describe separable locally compact groups which can be presented as continuous structures with a separably categorical continuous theory. The latter means that the group is determined uniquely (up to metric isomorphism) by its continuous theory among all separable groups.

Locally compact groups will be considered as one-sorted continuous structures in the continuous signature

$$
L=\left\{d, \cdot,^{-1}\right\}
$$

The research is supported by Polish National Science Centre Grant DEC2011/01/B/ST1/01406.

Aleksander Ivanov

iwanowaleksander@gmail.com

1 Institute of Mathematics, University of Wroclaw, pl. Grunwaldzki 2/4, 50-384 Wrocław, Poland

2 Institute of Mathematics, Silesian University of Technology, ul. Kaszubska 23, 44-100 Gliwice, Poland 
where $d$ denotes the metric. Let us recall that a metric $L$-structure is a complete metric space $(M, d)$ with $d$ bounded by 1 , where $\cdot$ and $^{-1}$ are uniformly continuous operations on $M$ [3]. It is assumed that to the symbols ${ }^{-1}$ and $\cdot$ continuity moduli $\gamma_{1}$ and $\gamma_{2}$ are assigned so that

$d\left(x_{1}, x_{2}\right)<\gamma_{1}(\varepsilon)$ implies $d\left(x_{1}^{-1}, x_{2}^{-1}\right)<\varepsilon$ and

$d\left(x_{1}, x_{2}\right)<\gamma_{2}(\varepsilon)$ implies $\max \left(d\left(x_{1} \cdot y, x_{2} \cdot y\right), d\left(y \cdot x_{1}, y \cdot x_{2}\right)\right)<\varepsilon$ for all $y \in M$.

Note that the latter condition is equivalent to existence of $\gamma_{3}(\varepsilon)$ (in fact $\gamma_{2}\left(\gamma_{2}(\varepsilon)\right)$ ) such that

$$
\left.d\left(x_{1}, x_{2}\right)<\gamma_{3}(\varepsilon) \text { implies } d\left(y \cdot x_{1} \cdot z, y \cdot x_{2} \cdot z\right)\right)<\varepsilon \text { for all } y, z \in M .
$$

We will also assume that the metric is left-invariant. We will see in Lemma 2 and Proposition 4 below that this assumption is natural.

We now state the main result of the paper. All logic notions appeared in the formulation and a proof of the theorem will be given in Sect. 2. We try to make it available for mathematicians outside model theory.

Theorem 1 Let $G$ be a locally compact group with a left-invariand metric $d \leq 1$ so that $(G, d)$ is a continuous metric structure. Then $(G, d)$ is a separably categorical metric structure if and only if there is a compact clopen subgroup $H<G$ which is invariant with respect to all metric automorphisms of $G$, and the induced action of Aut $(G, d)$ on the coset space $G / H$ is oligomorphic.

In this case if the connected component of the identity $G_{0}$ is a neighbourhood of the identity, $H$ can be taken to be $G_{0}$. When this happens or when $d$ is bi-invariant, the subgroup $H$ is normal and $G / H$ is an $\omega$-categorical discrete group.

We just remind the reader that an action of $G$ on a set $\Omega$ is called oligomorphic if for every $n$ the group $G$ has finitely many orbits on $\Omega^{n}$, see Section 2.1 in [5]. Concerning the second part of the statement note that in any Lie group the connected component $G_{0}$ is a neighbourhood of the identity (Section 3.0 in [11]). On the other hand to get an opposite example of a separably categorical group just take the direct sum of a countably categorical group with a compact $H$ such that $H_{0}$ is not open and $H_{0} \neq 1$. According to Corollary 5 below such a group has an appropriate metric.

In the rest of the introduction we discuss how powerful the continuous logic approach is in the class of locally compact groups. We start with the following lemma which shows that continuous logic can be applied only to metric groups which have bi-invariant metrics. This lemma appears as a part of Proposition 3.13 in [2].

Lemma 2 Let a group $(G, d)$ be a metric L-structure with respect to continuity moduli $\gamma_{1}$ and $\gamma_{2}$ as above. Then $G$ admits a complete bi-invariant metric $d^{*}$ which defines the same topology as $d$.

Proof We assume that $(G, d)$ is not discrete. Let $d^{*}(x, y)=\sup _{u, v} d(u \cdot x \cdot v, u \cdot y \cdot v)$. Then $d^{*}(x, y)$ is a bi-invariant metric with $d(x, y) \leq d^{*}(x, y)$. Since for every $\varepsilon$ we have

$$
d(x, y)<\gamma_{2}\left(\gamma_{2}(\varepsilon)\right) \Rightarrow d^{*}(x, y)<\varepsilon,
$$

each open $d$-ball contains an open $d^{*}$-ball and vice versa. 
Thus not all locally compact groups can be viewed as metric structures! In fact the continuous logic approach works only for SIN-groups.

Definition 3 ([9], Section 2) A topological group $G$ is called a SIN-group if any neighbourhood of the identity of $G$ contains a neighbourhood of the identity which is invariant under all inner automorphisms.

Proposition 4 A topological group $G$ admitting a complete metric can be presented as a continuous metric L-structure $(G, d)$ under d defining the topology of $G$ if and only if $G$ is a $\mathbf{S I N}$-group.

Proof V. Klee has shown in Section 1 of [10] that a Hausdorff group $G$ admits a biinvariant metric if and only if it admits a countable complete system of neighbourhoods of the identity which are invariant under all inner automorphisms. Thus a metrizable group $G$ has a bi-invariant metric if and only if $G$ is SIN. In this case we may assume that this metric $d$ is bounded by 1 (otherwise it can be replaced by $\frac{d(x, y)}{d(x, y)+1}$ ). In Section 2 of [10] it is shown that if a completely metrizable topological group $G$ has a bi-invariant metric then this metric is complete.

It is worth noting that a locally compact SIN-group is unimodular. Moreover SINgroups can be characterized among unimodular locally compact groups as those groups $G$ for which the von Neumann algebra $V N(G)$ generated by the left regular representation is finite (i.e. admits no non-unitary isometry), see [6], 13.10.5. Thus the class of locally compact bi-invariant metric groups still contains some interesting families (together with obvious examples: locally compact abelian groups and discrete groups). Moreover it is also known that Polish groups which are embeddable into the unitary group of a separable finite von Neumann algebra (i.e. the algebra has a faithful representation on a separable Hilbert space), admit complete bi-invariant metrics [13], Section 6.5 (also see Lemma 2.10 of [1]).

The following corollary of Theorem 1 and Lemma 2 gives a complete description of locally compact groups which can be presented as separably categorical metric structures. It substantially restricts the variety of examples mentioned above to ones which resemble semidirect products of countably categorical discrete groups with compact ones.

Corollary 5 A locally compact group $G$ can be presented as a separably categorical metric structure with respect to some metric if and only if there is a normal compact clopen subgroup $H<G$ with a bi-invariant metric d so that d is conjugacy invariant in $G$ and the group of automorphisms of $G / H$ which are induced by automorphisms of $G$ preserving $(H, d)$ is oligomorphic (in particular $G / H$ is a countably categorical discrete group).

The proof is given in Sect. 3. In Sect. 3 we also prove that when $G$ is separably categorical and $H$ is as in this statement, stability of the structure induced on $G / H$ is equivalent to stability of $G$ with respect to continuous formulas of some special type.

We finally note that although by Lemma 2 the metric $d$ in the formulation of Theorem 1 can be chosen bi-invariant, we do not assume this. The choice of $H$ may depend on the metric (see Remark 12).

The author is grateful to the referee for helpful remarks. 


\section{Preliminaries and the proof}

\subsection{Necessary preliminaries}

For convenience of the reader we recall some basic definitions from [3] and [4]. We keep the signature $L$ together with functions $\gamma_{1}$ and $\gamma_{2}$ as in Introduction. Then atomic formulas are the expressions of the form $d\left(t_{1}, t_{2}\right)$, where $t_{i}$ are terms (built from functional $L$-symbols). They take values from $[0,1]$. Statements concerning metric structures are formulated in the form

$$
\phi=0
$$

(called an L-condition), where $\phi$ is a formula, i.e. an expression built from numbers of $[0,1] \cap \mathbb{Q}$ and atomic formulas by applications of the following functions:

$$
\begin{aligned}
x / 2, x \dot{-} y & =\max (x-y, 0), \min (x, y), \max (x, y),|x-y|, \neg(x)=1-x, \\
x \dot{+} y & =\min (x+y, 1), x \cdot y, \sqrt{x}, \sup _{x} \text { and } i n f_{x} .
\end{aligned}
$$

A theory is a set of $L$-conditions without free variables (here $\sup _{x}$ and $i n f_{x}$ play the role of quantifiers). Formulas and statements are interpreted in continuous $L$-structures in the natural way. For simplicity we often replace expressions of the form $\phi \dot{-} \varepsilon=0$ with rational $\varepsilon$ by $\phi \leq \varepsilon$.

Let $M$ be a continuous metric $L$-structure. We define the automorphism group $\operatorname{Aut}(M)$ of $M$ to be the subgroup of $I \operatorname{so}(M, d)$ consisting of all isometries preserving the values of atomic formulas. It is easy to see that $A u t(M)$ is a closed subgroup with respect to the pointwise convergence topology on $I \operatorname{so}(M, d)$.

For every $c_{1}, \ldots, c_{n} \in M$ and $A \subseteq M$ we define the $n$-type $t p(\bar{c} / A)$ of $\bar{c}$ over $A$ as the set of all $\bar{x}$-conditions with parameters from $A$ which are satisfied by $\bar{c}$ in $M$. Let $S_{n}\left(T_{A}\right)$ be the set of all $n$-types over $A$ of the expansion of the theory $T$ by constants from $A$. There are two natural topologies on this set. The logic topology is defined by the basis consisting of sets of types of the form $[\phi(\bar{x})<\varepsilon]$, i.e. types containing some $\phi(\bar{x}) \leq \varepsilon^{\prime}$ with rational $\varepsilon^{\prime}<\varepsilon$. The logic topology is compact.

The $d$-topology is defined by the metric

$$
d(p, q)=\inf \left\{\max _{i \leq n} d\left(c_{i}, b_{i}\right) \mid \text { there is a model } N \text { with } N \models p(\bar{c}) \wedge q(\bar{b})\right\} .
$$

By Propositions 8.7 and 8.8 of [3] the $d$-topology is finer than the logic topology and $\left(S_{n}\left(T_{A}\right), d\right)$ is a complete space.

Definability in continuous structures is introduced as follows.

Definition 6 Let $M$ be a continuous metric $L$-structure and $A \subseteq M$. A uniformly continuous map $P: M^{n} \rightarrow[0,1]$ is called a predicate definable in $M$ over $A$ if there is a sequence $\left(\phi_{k}(\bar{x}): k \geq 1\right)$ of $L(A)$-formulas such that the maps interpreting $\phi_{k}(\bar{x})$ in $M$ converge to $P(\bar{x})$ uniformly in $M^{n}$. 
It is clear that a definable predicate defines a function on $S_{n}\left(T_{A}\right)$. By Propositions 3.4 and 3.10 of [4] functions $S_{n}\left(T_{A}\right) \rightarrow[0,1]$ which are continuous with respect to logic topology are precisely those given by definable predicates.

A tuple $\bar{a}$ from $M^{n}$ is algebraic in $M$ over $A$ if there is a compact subset $C \subseteq M^{n}$ such that $\bar{a} \in C$ and the distance predicate $\operatorname{dist}(\bar{x}, C)$ is definable in $M$ over $A$. Let $\operatorname{acl}(A)$ be the set of all elements algebraic over $A$. In continuous logic the concept of algebraicity is parallel to that in traditional model theory (see Section 10 of [3]).

A theory $T$ is separably categorical if any two separable models of $T$ are isomorphic. By Theorem 12.10 of [3] a complete theory $T$ is separably categorical if and only if for each $n>0$, every $n$-type $p$ is principal. The latter means that for every model $M \models T$, the predicate $\operatorname{dist}(\bar{x}, p(M))$ is definable over $\emptyset$.

Another property equivalent to separable categoricity states that for each $n>0$, the metric space $\left(S_{n}(T), d\right)$ is compact. In particular for every $n$ and every $\varepsilon$ there is a finite family of principal $n$-types $p_{1}, \ldots, p_{m}$ so that their $\varepsilon$-neighbourhoods cover $S_{n}(T)$.

In first order logic a countable structure $M$ is $\omega$-categorical if and only if $A u t(M)$ is an oligomorphic permutation group, i.e. for every $n, A u t(M)$ has finitely many orbits on $M^{n}$. In continuous logic we have the following modification.

Definition 7 An isometric action of a group $G$ on a metric space $(\mathbf{X}, d)$ is said to be approximately oligomorphic if for every $n \geq 1$ and $\varepsilon>0$ there is a finite set $F \subset \mathbf{X}^{n}$ such that

$$
G \cdot F=\{g \bar{x}: g \in G \text { and } \bar{x} \in F\}
$$

is $\varepsilon$-dense in $\left(\mathbf{X}^{n}, d\right)$.

Theorem 8 (C. Ward Henson, see Theorem 12.10 of [3] and Theorem 4.25 in [14]) Let $M$ be a separable continuous metric structure. The following conditions are equivalent.

(1) The theory of $M$ is separably categorical;

(2) The d-topology on $S_{n}$ coincides with the logic topology;

(3) The action of Aut $(M)$ on $(M, d)$ is approximately oligomorphic.

\subsection{Proof of Theorem 1}

The sufficiency follows from the description of separably categorical structures by approximate oligomorphicity of the group of their metric automorphisms. Indeed, given $n \geq 1$ and $\varepsilon>0$ choose a finite $\gamma_{2}(\varepsilon)$-net $F_{1}$ in the compact set of all $n$-tuples from $H$. Since $\operatorname{Aut}(G, d)$ is oligomorphic on $G / H$ there is a finite set $F_{2}$ of $n$-tuples from $G$ which represent all $\operatorname{Aut}(G, d)$-orbits on the set of all $n$-tuples from $G / H$. Then $\operatorname{Aut}(G, d) \cdot\left(F_{2} \cdot F_{1}\right)$ is $\varepsilon$-dense in the set of all $n$-tuples of $G$.

Let us prove the necessity of the theorem. We start with the following preliminaries. We may assume that $G$ is not discrete. There is a non-zero rational number $\rho<1$ such that the $\rho$-ball of the unity $B_{\rho}(e)=\{x \in G: d(x, e) \leq \rho\}$ is compact. Let $G_{\rho}$ 
be the subgroup generated by $B_{\rho}(e)$. Note that for any $g \in G_{\rho}$ the open ball

$$
B_{<\rho}(g)=\{x \in G: d(x, g)<\rho\}=\left\{x \in G: d\left(g^{-1} x, e\right)<\rho\right\}
$$

is a subset of $G_{\rho}$; thus $G_{\rho}$ is an open (in fact clopen) subgroup. If the connected component of the identity $G_{0}$ is a neighbourhood of the identity, we choose $\rho$ such that $B_{\rho}(e) \subseteq G_{0}$. Since $G_{0}$ is a subgroup of $G, G_{\rho} \leq G_{0}$. Since $G_{\rho}$ is open, $G_{\rho}=G_{0}$. Note that when $d$ is a bi-invariant metric, $G_{\rho}$ is a normal subgroup of $G$.

Lemma 9 Assume that $G$ is a separably categorical locally compact group. Then under the circumstances above the predicate $P(x)=d\left(x, G_{\rho}\right)$ is definable in $G$.

Proof Since $G_{\rho}$ is preserved under metric automorphisms of $G$ the predicate $P(x)$ naturally defines a map from the set of 1-types $S_{1}$ to $[0,1]$. Since $P(x)$ is continuous with respect to the $d$-topology on $S_{1}$, by separable categoricity it also continuous with respect to the logic topology. The latter exactly means that $P(x)$ is definable in $G$.

Remark Since $G_{\rho}$ is closed, this lemma states that $G_{\rho}$ is a definable set in the sense of continuous logic, see Definition 9.16 of [3].

Let us also note that since the condition $d(x, e) \leq \rho$ defines a totally bounded, complete subset in any elementary extension of $G$, the set $B_{\rho}(e)$ is a subset of $\operatorname{acl}(\varnothing)$ in $G$. Thus any $B_{\rho}^{n}(e)=B_{\rho}(e) \cdot B_{\rho}(e) \cdot \ldots \cdot B_{\rho}(e)$ also is a subset of $\operatorname{acl}(\emptyset)$. In particular $G_{\rho} \subset \operatorname{acl}(\emptyset)$.

Lemma 10 Under the circumstances above there is a natural number $n$ so that $G_{\rho}=$ $B_{\rho}^{n}(e)$. In particular $G_{\rho}$ is compact.

Proof Assume $G_{\rho} \neq B_{\rho}^{n}(e)$ for all $n \in \omega$. Notice that this implies that for every $n$ the $\rho$-neighbourhood of $B_{\rho}^{n}(e)$ does not cover $G_{\rho}$. Indeed if $g_{1} \in B_{\rho}^{n}(e), g_{2} \in G_{\rho}$ and $d\left(g_{1}, g_{2}\right)<\rho$, then $g_{1}^{-1} g_{2} \in B_{\rho}$ and $g_{2}=g_{1} g_{1}^{-1} g_{2} \in B_{\rho}^{n+1}$ (we use the assumption that $d$ is left-invariant). Thus if the $\rho$-neighbourhood of $B_{\rho}^{n}(e)$ covers $G_{\rho}$, then we have a contradiction with $G_{\rho} \neq B_{\rho}^{n+1}(e)$.

We see that the assumption $G_{\rho} \neq B_{\rho}^{n}(e)$ for all $n \in \omega$ implies that all statements

$$
\sup _{x_{1} \ldots x_{n}}\left(\min \left(\rho \dot{-} d\left(x, x_{1} \cdot \ldots \cdot x_{n}\right), \rho \dot{-} d\left(e, x_{1}\right), \ldots, \rho \dot{-} d\left(e, x_{n}\right)\right)\right)=0
$$

are finitely consistent together with $P(x)=0$. By Lemma 9 and compactness of continuous logic we obtain a contradiction.

Since $G_{\rho}$ is a characteristic subgroup of $G$ with respect to the automorphism group of the metric structure $G$, we see that $\operatorname{Aut}(G, d)$ acts correctly on $G / G_{\rho}$ by permutations of $G / G_{\rho}$. Note that $G / G_{\rho}$ is a discrete space with respect to the topology induced by the topology of $G$.

Lemma 11 The action of Aut $(G, d)$ on $G / G_{\rho}$ is oligomorphic.

Proof Since $(G, d)$ is separably categorical, $\operatorname{Aut}(G, d)$ is approximately oligomorphic on $(G, d)$. Thus for every $n$ there is a finite set $F$ of $n$-tuples from $G$ such that 
the set of orbits meeting $F$ is $\rho$-dense in $(G, d)$. In particular for any $g_{1}, \ldots, g_{n} \in G$ there is a tuple $\left(h_{1}, \ldots, h_{n}\right) \in F$ and an automorphism $\alpha \in \operatorname{Aut}(G, d)$ such that $g_{i}^{-1} \alpha\left(h_{i}\right) \in G_{\rho}$ for all $i \leq n$.

To see that Theorem 1 follows from lemmas above just take $H$ to be $G_{\rho}$.

Remark 12 We now illustrate that the choice of $H$ may depend on the metric. Let $G$ be

$$
S^{1} \times \mathbb{Z} / 2 \mathbb{Z} \times(\mathbb{Z} / 2 \mathbb{Z})^{\omega},
$$

where $S^{1}=\{z \in \mathbb{C}:\|z\|=1\}$ is the circle group and $\|x\|$ is the Euclidean norm in $\mathbb{C}$. Let us consider $G$ with respect to the metric $d_{1}$ defined as follows:

if $x_{1}$ and $x_{2}$ represent distinct $S^{1}$-cosets, then $d_{1}\left(x_{1}, x_{2}\right)=1$,

if $x_{1}$ and $x_{2}$ represent the same coset, then $d_{1}\left(x_{1}, x_{2}\right)=\frac{1}{4}\left\|1-x_{1}^{-1} x_{2}\right\|$.

Applying the proof of Theorem 1 for $\rho=1 / 2$ we obtain $H_{1}=G_{\rho}=B_{\rho}(e)=S^{1}$. Let us correct $d_{1}$ on the subgroup $S^{1} \times \mathbb{Z} / 2 \mathbb{Z}$ as follows:

if $x_{1}, x_{2} \in S^{1} \times \mathbb{Z} / 2 \mathbb{Z}$ represent distinct $S^{1}$-cosets, then $d_{2}\left(x_{1}, x_{2}\right)=\frac{1}{2}$,

if $x_{1}$ and $x_{2}$ represent the same coset, then $d_{2}\left(x_{1}, x_{2}\right)=\frac{1}{8}\left\|1-x_{1}^{-1} x_{2}\right\|$.

We obtain a bi-invariant metric $d_{2} \leq 1$. Applying the proof of Theorem 1 for $\left(G, d_{2}\right)$ and $\rho=1 / 2$ we see that the corresponding subgroup $H_{2}$ is different: $G_{\rho}=B_{\rho}(e)=$ $S^{1} \times \mathbb{Z} / 2 \mathbb{Z}$.

\section{Locally compact groups, separable categoricity and stability}

We start with the proof of Corollary 5:

A locally compact group $G$ can be presented as a separably categorical metric structure with respect to some metric if and only if there is a normal compact clopen subgroup $H<G$ with a bi-invariant metric $d$ so that $d$ is conjugacy invariant in $G$ and the group of automorphisms of $G / H$ which are induced by automorphisms of $G$ preserving $(H, d)$ is oligomorphic (in particular $G / H$ is a countably categorical discrete group).

In the proof of sufficiency we construct some canonical metric $d^{*}$ corresponding to the topology of $G$, so that $\left(G, d^{*}\right)$ is separably categorical. In the final part of the section we consider the question when $\left(G, d^{*}\right)$ is stable.

Proof of Corollary 5 If the locally compact group $G$ can be presented as a separably categorical metric structure with respect to some metric $\leq 1$ then by Lemma 2 (and its proof) we may choose an equivalent definable metric $d$ which is bi-invariant. Since 
the automorphism group of $(G, d)$ is still approximately oligomorphic, the structure $(G, d)$ is separably categorical. By Theorem 1 we find a normal compact clopen subgroup $H<G$ so that $G / H$ is an $\omega$-categorical discrete group.

To see the sufficiency of the theorem assume that $H<G$ is a compact, clopen normal subgroup. Choose a bi-invariant metric $d$ as in the formulation. We may assume that the $d$-diameter is $\frac{1}{2}$.

We consider the countable $\omega$-categorical group $G / H$ with respect to the $\{0,1\}$ metric, say $d_{0}$. Then consider $G$ with respect to so called wreath product of metrics $d^{*}=d_{0}$ wrt $d[12]$ :

if $x_{1}$ and $x_{2}$ represent distinct $H$-cosets, then $d^{*}\left(x_{1}, x_{2}\right)=1$,

if $x_{1}$ and $x_{2}$ represent the same coset, then $d^{*}\left(x_{1}, x_{2}\right)=d\left(1, x_{1}^{-1} x_{2}\right)$.

It is easy to see that $d^{*}$ is a metric. To see that it is a left-invariant metric assume that $x_{1}$ and $x_{2}$ represent the same coset with respect to $H$, and let

$$
x \cdot x_{i}=x_{i}^{\prime}, i \in\{1,2\} .
$$

Then $x_{1}^{-1} x_{2}=\left(x_{1}^{\prime}\right)^{-1} x_{2}^{\prime}$ and $d\left(x_{1}, x_{2}\right)=d\left(x_{1}^{\prime}, x_{2}^{\prime}\right)$. The case when $x_{i}$ do not represent the same coset is obvious. To see that $d^{*}$ is right-invariant apply the following argument.

We may suppose that $x_{1}$ and $x_{2}$ represent the same coset with respect to $H$. Let

$$
x_{i} \cdot x=x_{i}^{\prime}, i \in\{1,2\} .
$$

Then $x^{-1} x_{1}^{-1} x_{2} x=\left(x_{1}^{\prime}\right)^{-1} x_{2}^{\prime}$, i.e. $d\left(e, x^{-1} x_{1}^{-1} x_{2} x\right)=d\left(e,\left(x_{1}^{\prime}\right)^{-1} x_{2}^{\prime}\right)$ and since $d$ is conjugacy invariant we have $d\left(e, x_{1}^{-1} x_{2}\right)=d\left(e,\left(x_{1}^{\prime}\right)^{-1} x_{2}^{\prime}\right)$. In particular we see $d\left(x_{1}, x_{2}\right)=d\left(x_{1}^{\prime}, x_{2}^{\prime}\right)$.

To finish the proof note that since

$$
\phi\left(x_{1}\right)^{-1} \phi\left(x_{2}\right)=\phi\left(x_{1}^{-1} x_{2}\right), \text { where } \phi \in \operatorname{Aut}(G),
$$

any automorphism of $G$ which acts on $H$ as an automorphism of the continuous structure $(H, d)$, also preserves the metric $d^{*}$. Now the statement that $\left(G, d^{*}\right)$ is a separably categorical structure follows from sufficiency of Theorem 1.

Given $G$ as in the corollary is the group of automorphisms of $G / H$ which are induced by automorphisms of $G$ preserving $(H, d)$, closed in Aut $(G / H)$ ? By the final part of the proof above such automorphisms always extend to automorphisms of the continuous structure $\left(G, d^{*}\right)$. Having in mind this question let us consider $\operatorname{Aut}\left(G, d^{*}\right)$ as a metric group. We remind the reader that when $(\mathbf{Y}, d)$ is a Polish space the corresponding isometry group $I s o(\mathbf{Y})$ is a Polish group with respect to the pointwise convergence topology. A compatible left-invariant metric can be obtained as follows: fix a countable dense set $S=\left\{s_{i}: i \in\{1,2, \ldots\}\right\}$ and then define for two isometries $\alpha$ and $\beta$ of $\mathbf{Y}$ 


$$
\rho_{S}(\alpha, \beta)=\sum_{i=1}^{\infty} 2^{-i} \min \left(1, d\left(\alpha\left(s_{i}\right), \beta\left(s_{i}\right)\right)\right)
$$

Let us fix such a metric on $\operatorname{Aut}\left(G, d^{*}\right)$.

Lemma 13 The group of automorphisms of $G / H$ which are induced by automorphisms of $\left(G, d^{*}\right)$ is closed in Aut $(G / H)$.

Proof We denote by $\rho$ the metric $\rho_{S}$ which we have chosen for $\operatorname{Aut}\left(G, d^{*}\right)$. Here $S=\left\{s_{i}: i \in\{1,2, \ldots\}\right\}$ is the corresponding countable dense subset of $G$. Since $G$ is separable and $H$ is clopen, $G / H$ is countable and each $H$-coset contains an element of $S$. We order $G / H$ so that the number of any $g H$ is determined by the first representative of $g H$ from $S$. Note that the metric $\rho_{G / H}$ defined with respect to this enumeration of $G / H$ has the following presentation:

$$
\rho_{G / H}(\alpha, \beta)=\sum_{i=1}^{\infty}\left\{2^{-i}: \alpha \text { and } \beta \text { do not agree for the } i \text { th element of } G / H\right\}
$$

Let us consider a sequence $\alpha_{i} \in \operatorname{Aut}(G / H), i \in \omega$, converging to some $\alpha \in$ $\operatorname{Aut}(G / H)$ with respect to $\rho_{G / H}$. We assume that each $\alpha_{i}$ is induced by some $\gamma_{i} \in \operatorname{Aut}\left(G, d^{*}\right)$. Let us prove that there is a subsequence of $\left\{\gamma_{i}: i \in \omega\right\}$ converging to some $\gamma \in \operatorname{Aut}\left(G, d^{*}\right)$. We use the following construction.

At step $n>0$ we define a partial map $\delta_{n}$ on the $2^{-n}$-net $D_{n}$ of the first $n$ cosets of the enumeration of $G / H$ so that

(i) $D_{n}$ is contained in the minimal initial segment of $S$ which covers the first $n$ cosets of the enumeration of $G / H$ by a $2^{-n}$-net;

(ii) for any $s \in D_{n}$ we have $d^{*}\left(\delta_{n}(s), \delta_{n+1}(s)\right)<2^{-n-1}$ (this condition is empty for $n=0)$;

(iii) there is an infinite subsequence of $\alpha_{i}$ which agree with $\delta_{n}$ on the first $n$ cosets of $G / H$;

(iv) $\delta_{n}$ maps $D_{n}$ into the union of the cosets determined by $\operatorname{Im}\left(\alpha_{i}\right)$ as above and the subsequence in (iii) can be chosen so that for the corresponding $\gamma_{i}$ we have $d^{*}\left(\delta_{n}(s), \gamma_{i}(s)\right)<2^{-n-2}$ for all $s \in D_{n}$.

Let us assume that $D_{n-1}$ and $\delta_{n-1}$ are already defined. Extend $D_{n-1}$ to some $D_{n}$ so that (i) is satisfied. Take an infinite subsequence of $\alpha_{i}$ which

- agree with $\delta_{n-1}$ on the first $n-1$ cosets of $G / H$ and

- can be extended to a sequence of $\gamma_{i}$ satisfying

$$
d^{*}\left(\delta_{n-1}(s), \gamma_{i}(s)\right)<2^{-n-1} \text { for all } s \in D_{n-1} \text {. }
$$

We may also assume that all $\alpha_{i}$ agree at the $n$th coset of the enumeration of $G / H$. Since each $H$-coset is compact, we can find a finite $2^{-n-2}$-net, say $U_{n+2}$, in the union of all cosets from the image of the first $n$ cosets of $G / H$ with respect to our $\alpha_{i}$-s. Any $\gamma_{i}$ defines the map $D_{n} \rightarrow U_{n+2}$ which takes any $s \in D_{n}$ to the nearest element 
of $U_{n+2}$. Extending $U_{n+2}$ if necessary we may assume that these maps are injective. Since the set of these maps is finite we find an infinite subsequence of $\gamma_{i}$ defining the same map $D_{n} \rightarrow U_{n+2}$. We denote this map by $\delta_{n}$. Then condition (iv) is obvious and condition (ii) for $\delta_{n}$ follows from (iv) for $\delta_{n-1}$ and $\delta_{n}$ and the triangle inequality.

Having the sequence $\delta_{n}, n \in\{1,2, \ldots\}$, we choose $\gamma_{n}$ so that (iv) is satisfied for each pair $\delta_{n}$ and $\gamma_{n}$. Then for every initial segment of $G / H$ the sequence $\gamma_{n}$ is a Cauchy sequence of maps on the union of cosets of this segment with respect to the metric $\rho$. Thus the sequence $\gamma_{n}$ converges to some $\gamma$ in every union of this form.

From now on we consider $G / H$ under the structure induced by $\operatorname{Aut}\left(G, d^{*}\right)$, which exists by the lemma above.

We now discuss stability of $\left(G, d^{*}\right)$, where $d^{*}$ is constructed as in the proof of Corollary 5. Let us recall the following definition from Section 5.2 of [7].

A continuous theory $T$ has the order property if there is a formula $\psi(\bar{x}, \bar{y})$, where $\bar{x}$ and $\bar{y}$ are of the same length and sorts, and there is a model $M$ of $T$ with $\left(\bar{a}_{i}: i \in \omega\right) \subseteq M$, so that

$$
\psi\left(\bar{a}_{i}, \bar{a}_{j}\right)=0 \Leftrightarrow i<j \text { and } \psi\left(\bar{a}_{i}, \bar{a}_{j}\right)=1 \Leftrightarrow i \geq j .
$$

By compactness this condition is equivalent to the property that for all $n$ and $\delta \in(0,1)$ there are $\bar{a}_{1}, \ldots, \bar{a}_{n}$ such that

$$
\psi\left(\bar{a}_{i}, \bar{a}_{j}\right) \leq \delta \Leftrightarrow i<j \text { and } \psi\left(\bar{a}_{i}, \bar{a}_{j}\right) \geq 1-\delta \Leftrightarrow i \geq j
$$

The theory $T$ is called stable if it does not have the order property. In fact this coincides with the definition of stability given in Section 8 of [4].

We will concentrate on formulas $\psi$ which behave as slow functions.

Definition 14 Let $r \in[0,1]$. We will say that a continuous formula $\psi(\bar{x})$ is $r$-slow if there do not exist tuples $\bar{b}$ and $\bar{b}^{\prime}$ with $\max \left(d\left(b_{i}, b_{i}^{\prime}\right)\right) \leq r$ so that $\psi(\bar{b})=0$ and $\psi\left(\bar{b}^{\prime}\right)=1$.

In the situation of $\left(G, d^{*}\right)$, where $d^{*}$ is constructed as in the proof of Corollary 5 , if $b$ and $b^{\prime}$ represent the same cosets with respect to $H$ then the distance between them is $\leq 1 / 2$. In particular if $n$-tuples $\bar{b}$ and $\bar{b}^{\prime}$ represent the same cosets with respect to $H$ in an appropriate power $G^{k}$ then they are not distant at $>1 / 2$ under the corresponding max-metric defined by $d^{*}$. Thus in this situation a formula $\psi(\bar{x})$ is $\frac{1}{2}$-slow if and only if there do not exist tuples $\bar{b}$ and $\bar{b}^{\prime}$ which represent the same cosets with respect to $H$ so that $\psi(\bar{b})=0$ and $\psi\left(\bar{b}^{\prime}\right)=1$.

Theorem 15 Let $G$ be a locally compact group which can be presented as a separably categorical metric structure and let $H$ and $d^{*}$ be as in Corollary 5 and its proof.

Then the continuous theory $\operatorname{Th}\left(G, d^{*}\right)$ does not have $\frac{1}{2}$-slow formulas with the order property if and only if the elementary theory of the structure $G / H$ is stable.

Proof Assume that $\operatorname{Th}\left(G, d^{*}\right)$ has the order property witnessed by a $\frac{1}{2}$-slow formula $\psi(\bar{x}, \bar{y})$ and an $\omega$-sequence $\left(\bar{a}_{i}, i \in \omega\right)$. We know that $\psi$ is uniformly continuous. Thus replacing $\psi$ by appropriate roots $\psi^{2^{-l}}$ if necessary, we can arrange that for any 
tuples $\bar{b}_{1}, \bar{b}_{1}^{\prime}, \bar{b}_{2}$ and $\bar{b}_{2}^{\prime}$ of length $\left|\bar{a}_{i}\right|$ with $\max \left(\psi\left(\bar{b}_{1}, \bar{b}_{2}\right), d^{*}\left(\bar{b}_{1} \bar{b}_{2}, \bar{b}_{1}^{\prime} \bar{b}_{2}^{\prime}\right)\right)<1 / 2$ the value $\psi\left(\bar{b}_{1}^{\prime}, \bar{b}_{2}^{\prime}\right)$ is less than 1 . In particular defining $\psi(\bar{x}, \bar{y})$ on the discrete set $(G / H)^{k}$ by the minimal values on cosets corresponding to $\bar{x}$ and $\bar{y}$ we see that the relation $\psi(\bar{x}, \bar{y})<1 / 4$ has the order property on $G / H$ in the classical sense. Since it is invariant under the oligomorphic automorphism group of the structure on $G / H$ induced by $\operatorname{Aut}\left(G, d^{*}\right)$ we see that the theory of $G / H$ is not stable.

The opposite direction is easy. Having a relation $\theta(\bar{x}, \bar{y})$ with the order property with respect to the structure on $G / H$ we may define it on $G$ so that it takes constant values on cosets: 0 or 1 . Since the elements of distinct cosets are distant by 1 , we see that $\theta$ (viewed as a continuous formula) is continuous in $\left(G, d^{*}\right)$. It is also clear that it is $\operatorname{Aut}\left(G, d^{*}\right)$-invariant. Applying the argument of Lemma 9 we see that $\theta$ is definable as a continuous predicate (by a sequence of formulas). Take a close approximation of $\theta$, say $\theta^{\prime}$, which is a formula. If $\theta^{\prime}$ does not have values 0 or 1 , we can correct it as $2 \theta^{\prime}-\varepsilon$ for sufficiently small rational $\varepsilon$. As a result we obtain a $\frac{1}{2}$-slow formula with the continuous order property.

It is worth noting that the condition of $\frac{1}{2}$-slowness is essential in the theorem. For example an $\aleph_{0}$-categorical extra-special $p$-group $G$ has a finite definable subgroup $H$ so that $G / H$ is elementary abelian and thus is stable. On the other hand it is shown in [8] that extra-special $p$-groups are not stable. Our theorem shows that if we introduce appropriate $d^{*}$ (which defines the discrete topology in this case) the first-order formula defining the order property is not $\frac{1}{2}$-slow.

Open Access This article is distributed under the terms of the Creative Commons Attribution 4.0 International License (http://creativecommons.org/licenses/by/4.0/), which permits unrestricted use, distribution, and reproduction in any medium, provided you give appropriate credit to the original author(s) and the source, provide a link to the Creative Commons license, and indicate if changes were made.

\section{References}

1. Ando, H., Matsuzawa, Y.: On Polish groups of finite type. Publ. Res. Inst. Math. Sci. 48, 389-408 (2012)

2. Ben Yaacov, I.: Definability of groups in $\aleph_{0}$-stable metric structures. J. Symb. Log. 75, 817-840 (2010)

3. Ben Yaacov, I., Berenstein, A., Henson, W., Usvyatsov, A.: Model theory for metric structures. In: Chatzidakis, Z., Macpherson, H.D., Pillay, A., Wilkie, A. (eds.) Model theory with Applications to Algebra and Analysis, vol. 2. London Math. Soc. Lecture Notes, vol. 350, pp. 315-427, Cambridge University Press (2008)

4. Ben Yaacov, I., Usvyatsov, A.: Continuous first order logic and local stability. Trans. Am. Math. Soc. 362, 5213-5259 (2010)

5. Cameron, P.: Oligomorphic Permutation Groups. London Math. Soc. Lecture Notes, vol. 152, Cambridge University Press (1990)

6. Diximier, J.: Les $C^{*}$-algébres et leurs Représentations. Gauthier-Villas, Paris (1969)

7. Farah, I., Hart, B., Sherman, D.: Model theory of operator algebras II: model theory. Isr. J. Math. 201, 477-505 (2014)

8. Felgner, U.: On $\aleph_{0}$-categorical extra-special $p$-groups. Comptes Rendus de la Semaine d'Étude en Théorie des Modéles. Log. Anal. 18(71-72), 407-428 (1975)

9. Hofmann, K.H., Morris, S., Stroppel, M.: Varieties of topological groups, Lie groups and SIN groups. Coll. Math. 70, 151-163 (1996)

10. Klee Jr., V.L.: Invariant metrics in groups (solution of a problem of Banach). Proc. Am. Math. Soc. 3, 484-487 (1952) 
11. Montgomery, D., Zippin, L.: Topological Transformation Groups. Interscience Publishers, New York (1955)

12. Oliynyk, B.: Isometry groups of wreath products of metric spaces. Algebra Discrete Math. 4, 123-130 (2007)

13. Popa, S.: Cocycles and orbit equivalence superrigity for malleable actions of $w$-rigid groups. Invent. Math. 170, 243-295 (2007)

14. Schoretsanitis, K.: Fraïssé Theory for Metric Structures. PhD Thesis, University of Illinois at UrbanaChampaign (2007). http://www.math.uiuc.edu/ henson/cfo/metricfraisse 